\title{
Effect of antioxidant and optimal antimicrobial mixtures of carvacrol, grape seed extract and chitosan on different spoilage microorganisms and their application as coatings on different food matrices
}

\author{
Javiera F. Rubilar ${ }^{\mathrm{a}, \mathrm{c}}$, Rui M. S. Cruz ${ }^{\mathrm{b}, \mathrm{c}}$, Igor Khmelinskit ${ }^{\mathrm{a}, \mathrm{c}}$, and Margarida C. \\ VIEIRA $^{\text {b,c* }}$ \\ a Departamento de Química e Farmácia, Faculdade de Ciências e Tecnologia, Universidade do Algarve, Campus \\ de Gambelas, 8005-139 Faro, Portugal. \\ b Departamento de Engenharia Alimentar, Instituto Superior de Engenharia, Universidade do Algarve, Campus \\ da Penha, 8005-139 Faro, Portugal. \\ c Centro de Investigação em Química do Algarve (CIQA), Departamento de Química e Farmácia, Faculdade de \\ Ciências e Tecnologia, Universidade do Algarve, Campus de Gambelas, 8005-139 Faro, Portugal. E-mail \\ addresses: javiera.rubilar@gmail.com,rcruz@ualg.pt and ikhmelin@ualg.pt. \\ * Corresponding author \\ mvieira@ualg.pt \\ TEL: $+351-289-800900$ \\ FAX: $+351-289-800066$
}

Received: 14 March 2012; Published online: 18 April 2013

\begin{abstract}
There is growing interest in the use of natural agents with antimicrobial (AM) and antioxidant (AOX) properties. Optimization of the AM capacity for mixtures containing carvacrol, grape seed extract (GSE) and chitosan, against gram-negative (Pseudomonas aeruginosa), gram-positive bacteria (Staphylococcus aureus, Listeria innocua and Enterococcus faecalis) and yeast (Saccharomyces cerevisiae) at $10^{6} \mathrm{cfu} \mathrm{mL}^{-1}$ was studied. To observe the synergistic or antagonistic effect and find optimal combinations between the three agents, a simplex centroid mixture design was run for each microorganism, combining carvacrol (0-300 ppm, $\left.\mathrm{X}_{1}\right) \mathrm{X}$, GSE (0-2000 ppm, $\left.\mathrm{X}_{2}\right)$ and chitosan (0-2\% $\left.\mathrm{w} / \mathrm{v}, \mathrm{X}_{3}\right)$. Results of the response surface analysis showed several synergistic effects for all microorganisms. Combinations of $60 \mathrm{ppm}-400 \mathrm{ppm}-1.2 \% \mathrm{w} / \mathrm{v}$ (carvacrol-GSE-chitosan; optimal AM combination 1, OAMC-1); 9.6 ppm-684 ppm-1.25\% w/v (OAMC-2); 90 ppm-160 ppm-1.24\% w/v (OAMC-3) were found to be the optimal mixtures for all microorganisms. Radical scavenging activity (RSA) of the same agents was then compared with a standard AOX (butylated hydroxytoluene; BHT) at different concentrations (25, 50 and 100 ppm; as well as the optimal AM concentrations) by the 1,1-diphenyl-2picrylhydrazyl (DPPH) method. RSA increased in the following order: chitosan $<$ carvacrol $<\mathrm{BHT}<$ GSE and for the OAMC: OAMC-2< OAMC-1< OAMC-3. The best RSA (OAMC-3) was applied as a coating in two different food matrices (strawberries and salmon). For strawberries, $P$. aeruginosa was more sensitive to the action of OAMC-3 than $S$. cerevisiae. For salmon, S. aureus was more resistant to the action of OAMC-3 than E. faecalis and L. innocua.
\end{abstract}

Keywords: antimicrobial, antioxidant, carvacrol, chitosan, grape seed extract, coating, food. 


\section{Introduction}

Synthetic packaging films have led to serious ecological problems due to their nonbiodegradability. The high level of consumer concern for environmental protection led food and packaging industries to increase research in biodegradable food packaging materials (Fajardo et al., 2010). Biopolymers can be an alternative source for packaging materials' development due to their biodegradability. Chitin is an abundant naturally occurring biopolymer and is found in the exoskeleton of crustaceans, in fungal cell walls and in other biological materials. It is mainly $\operatorname{poly}(\beta$-(1-4)-2-acetamido-D-glucose), which is structurally identical to cellulose except that a secondary hydroxyl on the second carbon atom of the hexose repeat unit is replaced by an acetamide group (Geraldine, Ferreira Soares, Botrel, \& Goncalves, 2008). Chitosan is derived from chitin by deacetylation in an alkaline media. Therefore, chitosan is a copolymer consisting of $\beta$-(1-4)-2-acetamido-D-glucose and $\beta$-(1-4)-2amino-D-glucose units, with the latter usually exceeding $80 \%$. Moreover, chitosan is one of a few natural cationic polysaccharides, derived from crustacean or fungi, having antimicrobial (AM) properties against many bacteria, filamentous fungi and yeast (Rabea, Badawy, Stevens, Smagghe, \& Steurbaut, 2003). Furthermore, chitosan has antitumor and hypocholesterolemic functions but it does not have significant antioxidant (AOX) activity (Ojagh, Rezaei, Razavi, \& Hosseini, 2010). Several studies have shown that the AM and AOX effect of chitosan was greatly enhanced by the addition of essential oils (Jung et al., 2010). Carvacrol, the major component (50-86\%) of oregano (Origanum sp.), is a phenolic compound that has been used for many generations as a food flavouring agent and is generally recognized as safe (GRAS, as flavouring agent) by the FDA (US Food and Drug Administration, 2006). The AOX activity of carvacrol has been demonstrated (Aeschbach et al., 1994). Recently its AM activity against bacteria, mold and yeast has been reported, along with carvacrol's high potential to extend the shelf-life and improve safety of perishable foods (Guarda, Rubilar, Miltz, \& Galotto, 2011). Another compound known by its AM properties is grape seed extract (GSE). This is a rich source of monomeric phenolic compounds such as (+)-catechins, (-)-epicatechin, (-)-epicatechin3-o-gallate, gallic acid and dimeric, trimeric and tetrameric procyanidins, which have AOX (Kim \& Thomas, 2007) and antibacterial properties (Corrales, Han, \& Tauscher, 2009). The order of increasing AM activity reported for parts of the grape vine was flesh, whole fruit grape extracts, fermented fruit, fermented pomace, skin, leaves and seeds (Xia, Deng, Guo, \& Li, 2010).

Anastasiadi, Chorianopoulos, Nychas, and Haroutounian (2009) suggested that high concentrations of flavonoids and their derivatives in grape seed and of flavonoids, stilbenes and phenolic acids in grape stems were responsible for the AM activity. Rodriguez Vaquero, Alberto, and Manca de Nadra (2007) concluded that the non-flavonoid caffeic acid and the flavonoids such as rutin and quercetin have high inhibitory activities on growth of $L$. monocytogenes. Rhodes, Mitchell, Wilson, and Melton (2006) demonstrated that polymeric phenolic fractions produced the highest inhibition activity for all Listerial species, but not for other bacteria such as B. cereus, Salmonella menston, E. coli, S. aureus or Yersinia enterocolitica.

On the other hand, chitosan's application as an edible film and coating is very attractive, and it has been reported in several studies as one of the most promising coating materials for different food matrices (Park, Stan, Daeschel, \& Zhao, 2005; Park \& Zhao, 2004). However, none have studied optimal combinations of chitosan and natural AM agents. The present study aims to develop an optimal mixture of carvacrol, GSE and chitosan for the growth inhibition of five different microorganisms, and test its usage in two different food matrices (strawberries and salmon), thereby contributing to future applications in coatings and active packaging films with AM and AOX properties. 
Effect of carvacrol, grape seed extract and chitosan mixtures on microorganisms and food $\mid 23$

\section{Materials and Methods}

\subsection{Microorganisms and media}

Listeria innocua (ATCC 33090), Staphylococcus aureus (ATCC 25923), Enterococcus faecalis (ATCC 29212), Pseudomonas aeruginosa (ATCC 27853) and Saccharomyces cerevisiae (ATCC 9763) were obtained from Iberlab\&imunoreage ${ }^{\circledR}$ (Lisbon, Portugal). Yeast malt agar (YMA, for yeast), tryptic soy broth (TSB, for bacteria), tryptic soy agar (TSA, for bacteria), and tryptone water were obtained from Scharlau (Lisbon, Portugal). Yeast malt broth (YMB, for yeast) was obtained from Merck (Lisbon, Portugal).

\subsection{Antimicrobial agents and chemicals}

Carvacrol (98\% pure; Cat: 28,219-7), 1,1diphenyl-2-picrylhydrazyl (DPPH; Cat: D9132$1 \mathrm{G}$ ), butylated hydroxytoluene (BHT; Cat: 47168) and chitosan (high molecular weight; with a deacetylation degree of $>75 \%$, Cat: 419419250G) were obtained from Sigma Aldrich (Lisbon, Portugal). Grape seed extract (GSE; exGrape ${ }^{\circledR}$ seed OPC 40 powder, polyphenols $>95 \%$ and proanthocyanidins $>70 \%$ ) was obtained from Gardonnenque (Groupe Grap'Sud, Cruviers Lascours, France). Ethanol and acetic acid were purchased from Merck (Lisbon, Portugal). Methanol was obtained from José Manuel Gomes dos Santos (JMGS Odivelas, Portugal).

\subsection{Determination of carvacrol and GSE minimal inhibitory concentrations}

The minimal inhibitory concentrations (MIC) of carvacrol and GSE were determined for the five studied microorganisms (L. innocua, S. aureus, E. faecalis, $P$. aeruginosa and $S$. cerevisiae). Stock solutions of the AM agents were prepared, based on previous studies by Jayaprakasha, Selvi, and Sakariah (2003) and Guarda et al. (2011), as follows: $30 \%$ of carvacrol in ethanol and $10 \%$ of GSE in distilled water. Serial dilutions of carvacrol $(7500 ; 6000 ; 4500 ; 3000 ; 1500 ; 750 ; 375$;
$300 ; 225 ; 150 ; 75$ and $37.5 \mathrm{ppm})$ and GSE (16000, 12000, 8000, 4000, 2000, 1000, 500, 250, 125 ppm) were prepared in sterile TSA for the bacteria and in sterile YMA for the yeast. Petri dishes were inoculated with $1 \mathrm{~mL}$ of $10^{6} \mathrm{cfu}$ for bacteria (growth in TSB for $18 \mathrm{~h}$ at $37^{\circ} \mathrm{C}$ ) and yeast (growth in $\mathrm{YMB}$ for $18 \mathrm{~h}$ at $28^{\mathrm{O}} \mathrm{C}$ ) (except $\mathrm{P}$. aeruginosa where an inoculum of $104 \mathrm{cfu} \mathrm{mL}^{-1}$ was also tested) and incubated (Binder, BD-115, Germany) at $37^{\circ} \mathrm{C}$ (bacteria) and $28{ }^{\circ} \mathrm{C}$ (yeast) for $48 \mathrm{~h}$. The lowest concentration able to inhibit visible growth (colony-forming units (cfu) on the surface of the solid medium) of the test microorganisms was taken as being the MIC Guarda et al. (2011). The highest concentration of ethanol (used as solvent for carvacrol) was used as a control, to verify that ethanol had no AM effect. All experiments were run in triplicate.

\subsection{Experimental Design}

Response surface methodology was used in order to test the synergies between the three compounds (carvacrol, GSE and chitosan). The MIC obtained for $L$. innocua and E. faecalis were selected, which were $300 \mathrm{ppm}$ for carvacrol, and 2000 ppm for GSE. A simplex centroid mixture (SCM) design was run for each microorganism using the Design-Expert ${ }^{\circledR} 7.0$ software (DesignExpert software, 2005), at $300 \mathrm{ppm}$ carvacrol, $2000 \mathrm{ppm}$ GCE and $2 \% \mathrm{w} / \mathrm{v}$ chitosan, with the objective to find one or more optimal mixtures with a broad spectrum of action and effective simultaneously against yeast, gram-positive and gram-negative bacteria. The above MIC values were selected, because the other MIC were either too high, as in the case of $P$. aeruginosa, showing values of MIC greater than $7500 \mathrm{ppm}$ for carvacrol and values of MIC of $16000 \mathrm{ppm}$ for GSE, or too low, such as $S$. cerevisiae or $S$. aureus, showing MIC values of 150 and 225 ppm for carvacrol, respectively. Therefore, we selected the values close to the average MIC values obtained for the five microorganisms tested.

In order to check the viability of all bacteria and yeast, a control was run with TSA for bacteria and YMA for yeast.

The data obtained from the SCM design were fitted with a third order polynomial equation (Eq. 
1). The equation was as follows:

$$
\begin{aligned}
& Y_{i}=\beta_{1} x_{1}+\beta_{2} x_{2}+\beta_{3} x_{3}+\beta_{12} x_{1} x_{2} \\
& +\beta_{23} x_{2} x_{3}+\beta_{13} x_{1} x_{3}+\beta_{123} x_{1} x_{2} x_{3}
\end{aligned}
$$

where $\mathrm{Y}_{i}$ is the response variable of the evaluated parameter i (defined as log reduction for each microorganism, which was the difference between what grew in the agar control and what grew in the agar with added AM solutions). Prediction models were obtained for $\mathrm{Y}_{1}$ : $\log$ reduction of L. innocua; $\mathrm{Y}_{2}$ : $\log$ reduction of $S$. aureus; $\mathrm{Y}_{3}$ : $\log$ reduction of $E$. faecalis; $\mathrm{Y}_{4}$ : $\log$ reduction of $P$. aeruginosa and $\mathrm{Y}_{5}$ : $\log$ reduction of $S$. cerevisiae; where $\beta_{1}, \beta_{2}, \beta_{3}, \beta_{12}, \beta_{23}, \beta_{13}$ and $\beta_{123}$ are the estimated coefficients (Myers \& Montgomery, 1995). The different combinations of the three natural agents, carvacrol (0-300 ppm, $\left.\mathrm{X}_{1}\right)$, GSE (0-2000 ppm, $\left.\mathrm{X}_{2}\right)$ and chitosan $(0-2 \% \mathrm{w} / \mathrm{v}$, $\left.\mathrm{X}_{3}\right)$, were prepared following the proportions determined by the experimental design (Table 1 ), in sterile TSA for bacteria and sterile YMA, for yeast, according to the method of Guarda et al. (2011). The plates inoculated with $10^{6} \mathrm{cfu} \mathrm{mL}^{-1}$ of each of the microorganisms were incubated at the appropriate conditions (section 2.3). The adequacy of the model was determined by evaluating the coefficient of determination $\left(\mathrm{R}^{2}\right)$ and the Fisher test value ( $F$-value) obtained from the analysis of variance (ANOVA). Statistical significance of the model and model variables were determined at a significance level of 0.05. The cubic model equation was used by the software to build the response surface. Three-dimensional response surface plots were generated by keeping one response variable at its optimal level and plotting that against three factors (independent variables). The complete design for each microorganism consisted of 18 experimental points including three replications of the central point (Fig. 1). The actual values of the factors for the experimental design are given in Table 1.

\subsection{Optimization and model validation}

The goal (maximum logarithmic reduction) and constraint (six logarithmic reductions at most) were established for each response in order to

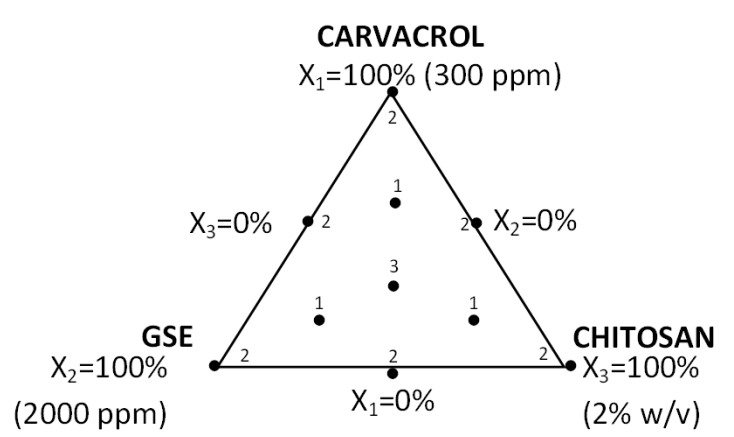

Figure 1: Simplex centroid mixture design.

carry out an optimization between the three natural AM agents for each microorganism and to select the best possible combination. Several researchers reported concentrations of chitosan solutions between $1 \%$ and $2 \% \mathrm{w} / \mathrm{v}$ for the development of chitosan films (Pranoto, Rakshit, \& Salokhe, 2005; Durango et al., 2006). Therefore, the percentage of chitosan for optimization was restricted between $60 \%(1.2 \% \mathrm{w} / \mathrm{v})$ and $70 \%$ $(1.4 \% \mathrm{w} / \mathrm{v})$. An experimental validation was performed in order to confirm the optimal solutions selected by the prediction models for each microorganism. The experimental validation was run in triplicate.

\subsection{Antioxidant activity (DPPH radical assay)}

The measurements of the free radical scavenging effect of carvacrol, GSE and chitosan were based on previous studies by Sokmen et al. (2004), Baydar, Ozkan, and Yasar (2007), and Kim and Thomas (2007) and compared to BHT, a known antioxidant, by the DPPH method. A $1.0 \mathrm{~mL}$ solution of the agents at different concentrations $(25,50,100$ ppm as well as the optimal AM concentrations OAMC-1, OAMC-2 and OAMC-3) in methanol was mixed with $2.0 \mathrm{~mL}$ of methanolic solution of DPPH $\left(40 \mathrm{mg} \mathrm{L}^{-1}\right)$. The mixture was shaken vigorously and allowed to stand at room temperature for $30 \mathrm{~min}$. Then, the absorbance was measured at $517 \mathrm{~nm}$ against methanol (blank) in a spectrophotometer. Lower absorbance of the reaction mixture indicated 
Effect of carvacrol, grape seed extract and chitosan mixtures on microorganisms and food $\mid 25$

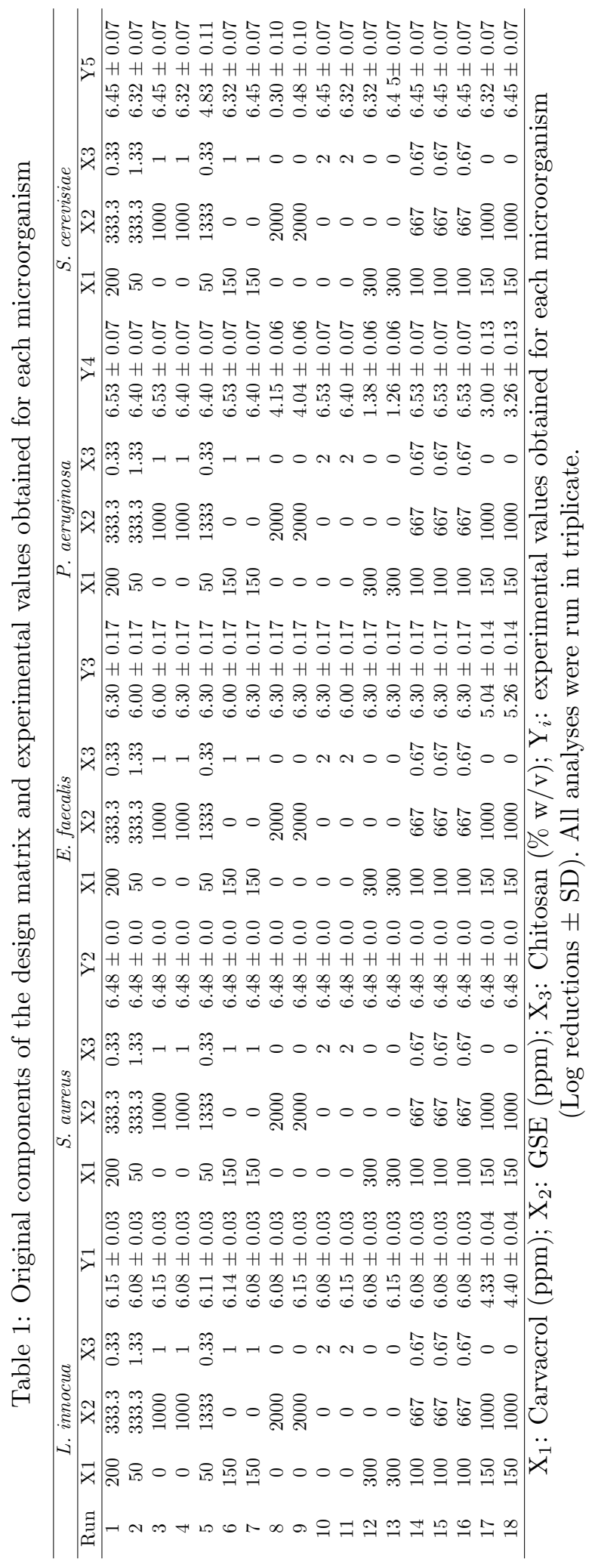

IJFS | April 2013 | Volume 2 | pages 22-38 
higher free radical scavenging activity. All determinations were carried out in triplicate.

The percent of DPPH decoloration of the samples was calculated according to the formula (Eq. 2).

$$
\text { Antiradical activity }=100 \times\left(1-\left(\frac{\text { Sample absorbance }}{\text { Control absorbance }}\right)\right)
$$

\subsection{Inoculation and coating treatment of strawberry and salmon}

Fresh strawberries were purchased from a local supermarket immediately after they arrived from storage. Fruits were selected for uniform size, diameter, colour and maturity (3/4 of surface showing red) and for being free of visible defects and decay. The strawberries were dipped into $250 \mathrm{ppm}$ sodium hypochlorite for $30 \mathrm{~s}$ and rinsed with distilled water. No residual chlorine was ensured with a control test. It is known that chlorine in contact with salmon (or organic matter) can produce organic chlorinates (carcinogenic), nevertheless, to reproduce the same conditions for salmon and strawberries, the experiment was performed with chlorine. Fresh salmon was purchased from a local seafood market, and stored for a short period of time. Salmon was cut into pieces with the following dimensions: approximately $3 \mathrm{~cm} \mathrm{x} 3 \mathrm{~cm} \times 1.5 \mathrm{~cm}$ (length, width and thickness).

The methodology for salmon and strawberry was based on a previous study with modifications (Park et al., 2005). Strawberries were inoculated with $10^{6} \mathrm{cfu} \mathrm{mL}^{-1}$ suspensions of $S$. cerevisiae and $P$. aeruginosa (separately) by dipping strawberries in $10^{6} \mathrm{cfu}$ suspension for one min and dried on foil for $1 \mathrm{~h}$ before subjecting to coating application. Salmon samples were inoculated with $10^{6} \mathrm{cfu} \mathrm{mL}^{-1}$ suspensions of $L$. innocua, E. faecalis and $S$. aureus (separately) by dipping each salmon piece in $10^{6} \mathrm{cfu}$ suspensions for one min and dried on foil for $1 \mathrm{~h}$ before coating application.

Inoculated strawberries and salmon pieces were coated with the solution with the best AOX capacity (optimal AM combination 3; OAMC-3), and two controls were also analysed (dipped in distilled water and residual chlorine). Strawberries and salmon pieces were dipped in coating solutions at room temperature $\left(22^{\circ} \mathrm{C}\right)$ for $1 \mathrm{~min}$ and dried on a sterile stainless-steel screen with $70 \%$ ethanol under fans $\left(2.2 \pm 0.2 \mathrm{~m} \mathrm{~s}^{-1}\right)$ for about $1 \mathrm{~h}$ to ensure surface dryness. Fruits were then packed in a sterile expanded polystyrene food tray, which was placed within a polyethylene bag, and stored at refrigeration temperature

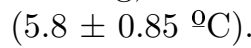

\subsection{Microbiological analysis}

Microbiological analysis was carried out on days 0 and 2. After 16 days visual difference between the control and coated food matrices (strawberries and salmon) were observed.

$10 \mathrm{~g}$ of inoculated and coated food matrix (strawberries and salmon) were mixed with $90 \mathrm{~mL}$ of tryptone water for $120 \mathrm{~s}$ in a Stomacher bag (Seward, Stomacher 400), using a Stomacher blender (Seward, Stomacher 400, London, England). The suspension was plated with appropriate dilutions on YMA for yeast and TSA for bacteria. YMA plates were incubated for $48 \mathrm{~h}$ at $28{ }^{\circ} \mathrm{C}$ and TSA for $48 \mathrm{~h}$ at $37^{\circ} \mathrm{C}$. The experiments were run in triplicate.

\subsection{Statistical analysis}

A randomized experimental design was used. Data analyses were carried out using Statgraphics Centurion XV software (2006), based on analysis of variance and the Student's $t$-test. Differences were considered at a significance level of $\alpha=0.05$.

\section{Results and Discussion}

\subsection{Carvacrol and GSE minimal inhibitory concentrations}

For carvacrol the density was considered to be $0.976 \mathrm{~g} \mathrm{~mL}^{-1}$, at $20{ }^{\circ} \mathrm{C}$, obtaining concentrations of $50 ; 40 ; 30 ; 20 ; 10 ; 5 ; 2.5 ; 2.0 ; 1.5 ; 1.0 ; 0.5$ and $0.25 \mathrm{mmol}$. For comparison purposes with GSE, the concentrations of carvacrol were converted into ppm. 
Effect of carvacrol, grape seed extract and chitosan mixtures on microorganisms and food $\mid 27$

Carvacrol and GSE exhibited AM activity against most microorganisms tested in this study. P. aeruginosa was the most resistant to the action of carvacrol showing values of MIC greater than $7500 \mathrm{ppm}\left(10^{6} \mathrm{cfu} \mathrm{mL}^{-1}\right)$. L. innocua and E. faecalis, presented values of MIC of 300 ppm and $S$. aureus a MIC of $225 \mathrm{ppm}$, showing less resistance to the action of this natural AM agent than $P$. aeruginosa. The yeast was the least resistant to the action of carvacrol presenting a MIC of $150 \mathrm{ppm}$. These results are similar to those found by Cosentino et al. (1999) who tested the AM activity of Thymus essential oils and found that yeasts were the most sensitive among the studied microorganisms, followed by the gram-positives and coming last the gramnegative bacteria. The AM activity of essential oils, which contain high percentages of carvacrol, has been studied by different researchers. These compounds have shown to be inhibitory on selected food-spoilage organisms (Sivropoulou et al., 1996; Smith-Palmer, Stewart, \& Fyfe, 1998; Cosentino et al., 1999; Hammer, Carson, \& Riley, 1999). Most researchers have reported that the gram-positive bacteria are more sensitive than gram-negative bacteria when using different essential oils (Marino, Bersani, \& Comi, 2001; Pintore et al., 2002). This is in agreement with the results of the present study, regarding the effect of carvacrol on $P$. aeruginosa. Hence, it was essential to determine the carvacrol MIC for a lower inoculum. A MIC of $1500 \mathrm{ppm}$ of carvacrol was obtained for $10^{4} \mathrm{cfu} \mathrm{mL}^{-1}$ of $P$. aeruginosa. Sivropoulou et al. (1996) and Hammer et al. (1999) determined the AM activity of an extract from Origanum vulgare containing carvacrol ( $80 \%$ ), and found that $P$. aeruginosa presented a MIC of $2000 \mathrm{ppm}$, a result which was similar to those of other authors (Cosentino et al., 1999; Lambert, Skandamis, Coote, \& Nychas, 2001). The higher resistance of gram-negative bacteria to the action of the AM agents can be explained by the outer membrane that surrounds the lipopolysaccharides (LPS) wall in these microorganisms, thereby restricting the diffusion of hydrophobic compounds (Vaara, 1992).

Carvacrol is able to disintegrate the outer membrane of the gram-negative bacteria, releasing the LPS, which may increase the permeability of the adenosine triphosphate in the cytoplasmic membrane, and consequently change the passive permeability of the cell. Therefore, a higher concentration of the AM agent is needed to produce the same effect as in the gram-positive bacteria (Smith-Palmer et al., 1998; Burt, 2004; ŠegvićKlarić, Kosalec, Mastelić, Piecková, \& Pepeljnak, 2006).

Burt (2004) reviewed several studies, in which essential oils and/or active compounds such as carvacrol were analysed, and found MICs for $S$. aureus and Listeria spp. very similar to those reported in this study. Veldhuizen, TjeerdsmaVan Bokhoven, Zweijtzer, Burt, and Haagsman (2006) studied the inhibitory effect of carvacrol and carvacrol-related compounds against $S$. aureus. Carvacrol presented a MIC of $225 \mathrm{ppm}$ (very similar to this study) and was more effective than carvacrol-related compounds. The GSE showed lower AM activity than carvacrol against all microorganisms. The yeast $(S$. cerevisiae) was the most resistant to the action of this AM agent (no inhibitory effect) presenting a MIC exceeding $16000 \mathrm{ppm}$. This polyphenolic resistance can be explained by the yeast natural environment (e.g. grapes and wines) in which several phenolic compounds are present. For this reason, many authors only determined the antibacterial capacity of GSE (Jayaprakasha et al., 2003; Baydar, Ozkan, \& Sagdic, 2004; Baydar, Sagdic, Ozkan, \& Cetin, 2006; Anastasiadi et al., 2009). The gram-positive bacteria presented a MIC of 2000 ppm for L. innocua and E. faecalis, and a MIC of $500 \mathrm{ppm}$ for S. aureus. P. aeruginosa was more resistant to the action of GSE than the gram-positive bacteria, as reported in other studies (Jayaprakasha et al., 2003; Baydar et al., 2004; Baydar et al., 2006). The P. aeruginosa yielded MIC of $4000 \mathrm{ppm}\left(10^{4} \mathrm{cfu} \mathrm{mL}^{-1}\right)$

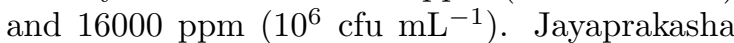
et al. (2003) determined the antibacterial capacity of GSE and found a MIC of $1000 \mathrm{ppm}$ for S. aureus. Shoko et al. (1999) have also reported the AM activity of GSE and proposed that gallic acid was the active compound responsible for the inhibition of E. coli and Salmonella enteritidis. Thuille, Fille, and Nagl (2003) studied the inhibitory effect of grape kernel extract against several pathogens and presented MICs of 1000 ppm and $1500 \mathrm{ppm}$ for $S$. aureus and P. aeruginosa, respectively. The bacterial growth inhi- 
bition caused by GSE can be described by several mechanisms of action. Namely, polyphenols can penetrate the semipermeable bacterial membrane, reacting with the cytoplasm or cellular proteins. This potential is higher in GSE as phenolic acids are present in an undissociated form (Paulus, 1993; Corrales et al., 2009).

Scalbert (1991) proposed that the antibacterial activity of tannins, compounds present in GSE, could be due to the inhibition of extracellular microbial enzymes. The resistance of gram-negative bacteria to GSE can be explained by their lipidic wall, a strong barrier against polyphenols' action. Different extraction procedures and grape seed types may explain the different AM activities achieved in different studies (Corrales et al., 2009).

\subsection{Response prediction models}

The grades given for each response were averaged for each formulation, defined by the experimental design (Table 1). Through statistical analysis (Design-Expert, 2005), a search for the prediction model that could best fit the experimental data for each response was then performed. Analysis of sequential F-test, for each response, indicate whether the cubic terms $\beta_{123} \mathrm{X}_{1} \mathrm{X}_{2} \mathrm{X}_{3}$ would contribute significantly (at $5 \%$ level) to the model or not. The model fitting analysis (Table 2) showed, for all the studied microorganisms, a prediction coefficient of determination in reasonable agreement with the adjusted coefficient of determination. The precision with a ratio greater than 4 was also obtained for all models for each microorganism. Therefore, all models could be used to navigate the design spaces. Prediction models were obtained for all responses $\mathrm{Y}_{1}, \mathrm{Y}_{2}$, $\mathrm{Y}_{3}, \mathrm{Y}_{4}$ and $\mathrm{Y}_{5}$ as shown in Table 3.

Response surfaces are presented in Fig. 2 for each microorganism. L. innocua and E. faecalis presented a very similar response surface, at low values of carvacrol and GSE and high chitosan concentration (red surface, Fig. 2). For S. aureus, all combinations showed 6 log reductions because the MICs of carvacrol and GSE were 225 ppm and $500 \mathrm{ppm}$, respectively. Therefore, this result was expected because $S$. aureus was the microorganism more sensitive to the action of
$\mathrm{AM}$ agents and the concentrations used in this mixture design were higher in all combinations of carvacrol, GSE and chitosan than the MIC for S. aureus.

The concentration used for $P$. aeruginosa was lower than the corresponding MICs (>7500 for carvacrol and $16000 \mathrm{ppm}$ for GSE) but the result, as can be seen in the Fig. 2, was synergistic (concentration below the MICs), obtaining $6 \log$ reductions near to the chitosan zone. The response surface for the $S$. cerevisiae clearly shows that the maximum logarithmic reduction was close to the maximum values of carvacrol and chitosan, and no inhibition was found near the maximum values of GSE due to the polyphenolic compounds that are a natural environment for this type of yeast.

All plots in Fig. 2, except P. aeruginosa, showed that concentrations of carvacrol lower than 300 ppm (100\% carvacrol) and concentrations of GSE lower than $2000 \mathrm{ppm}(100 \%$ GSE), in mixture with chitosan (lower than $2 \% \mathrm{w} / \mathrm{v}$ ), are sufficient for obtaining six logarithmic reductions for each of the studied microorganisms. The largest logarithmic reduction zone, resulting from a synergistic effect, was observed at $50 \%$ of carvacrol, $50 \%$ of GSE and high values of chitosan.

\subsection{Optimization and model validation}

Several optimal AM combinations (OAMC) were obtained by the program, but only three were selected for all microorganisms. One of the OAMCs selected contained the highest percentage of GSE (OAMC-1, in Table 4), another contained similar proportions between carvacrol and GSE (OAMC-2, in Table 4) and the third contained the highest percentage of carvacrol (OAMC-3, in Table 4).

The three optimal combinations obtained between the three natural agents (carvacrol, GSE and chitosan solution) in the models for all the microorganisms (Table 4) were validated experimentally. The experimental validation showed that the same optimal combination between the three AM agents, selected by the models, can produce six logarithmic reductions for all of the studied microorganisms. 
Effect of carvacrol, grape seed extract and chitosan mixtures on microorganisms and food $\mid 29$

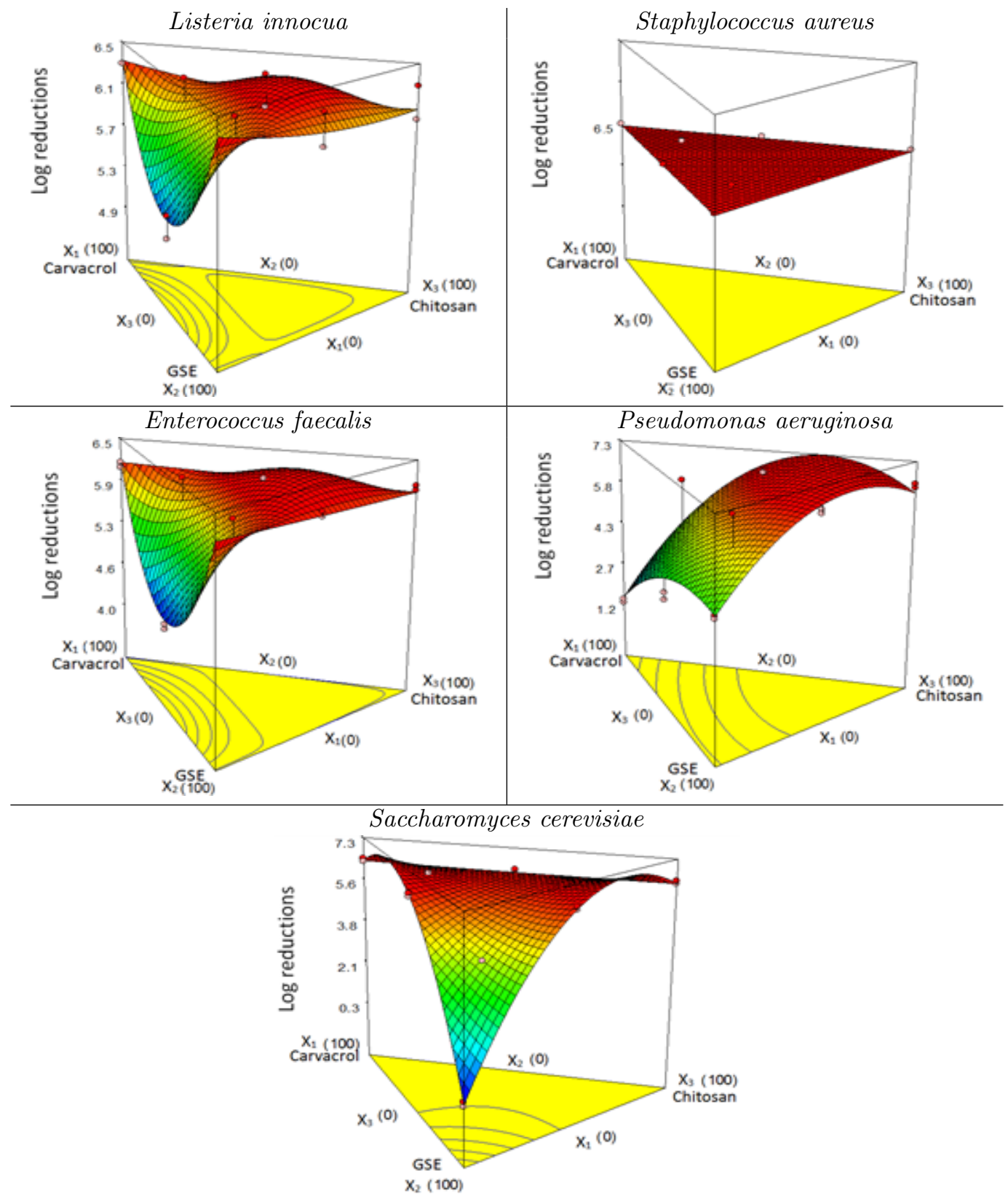

Figure 2: Response surfaces for the effect of carvacrol, GSE and chitosan on the log reduction of different microorganisms $\left(\mathrm{X}_{1}\right.$ : Carvacrol, $\mathrm{X}_{2}$ : GSE and $\mathrm{X}_{3}$ : Chitosan). 
Table 2: Summary of the analysis of variance for all responses.

\begin{tabular}{|c|c|c|c|c|c|c|c|c|c|c|c|c|c|c|c|}
\hline Source & \multicolumn{3}{|c|}{$\begin{array}{l}\text { Log Reductions } \\
\text { L. inпосиа }\end{array}$} & \multicolumn{3}{|c|}{$\begin{array}{c}\text { Log Reductions } \\
\text { S. aureus }\end{array}$} & \multicolumn{3}{|c|}{$\begin{array}{c}\text { Log Reductions } \\
\text { E. faecalis }\end{array}$} & \multicolumn{3}{|c|}{$\begin{array}{c}\text { Log Reductions } \\
\text { P. aeruginosa }\end{array}$} & \multicolumn{3}{|c|}{$\begin{array}{l}\text { Log Reductions } \\
\text { S. cerevisiae }\end{array}$} \\
\hline Model & & pecial C & & Linear & & & & ecial c & bic & & ecial C & bic & & ecial C & bic \\
\hline & $\mathrm{df}$ & MS & F-value & $\mathrm{df}$ & MS & F-value & $\mathrm{df}$ & MS & F-value & df & MS & F-value & $\mathrm{df}$ & MS & F-value \\
\hline Model & 6 & 0.84 & $22.93^{*}$ & 2 & - & - & 6 & 0.32 & $7.66^{*}$ & 6 & 8.69 & $15.60^{*}$ & 6 & 10.52 & $412.58^{*}$ \\
\hline Residual & 9 & 0.036 & & 13 & - & & 9 & 0.041 & & 9 & 0.56 & & 9 & 0.025 & \\
\hline Lack of Fit & 3 & 0.10 & $44.36^{*}$ & & & & 3 & 0.071 & 2.69 & 3 & 1.65 & 136.02 & 3 & 0.058 & 6.42 \\
\hline Pure Error & 6 & 0.0024 & & 6 & - & & 6 & 0.026 & & 6 & 0.012 & & 6 & 0.010 & \\
\hline Corrected Total & 15 & & & 15 & & & 15 & & & 15 & & & 15 & & \\
\hline $\mathrm{R}_{a d j}^{2}$ & 0.8977 & & & - & & & 0.7271 & & & 0.8538 & & & 0.9940 & & \\
\hline $\mathrm{R}_{\text {pred }}^{2}$ & 0.8599 & & & - & & & 0.5642 & & & 0.8113 & & & 0.9883 & & \\
\hline Adeq. Prec. & 14.998 & & & - & & & 8.515 & & & 11.958 & & & 58.883 & & \\
\hline Std. Dev. & 0.19 & & & - & & & 0.20 & & & 0.75 & & & 0.16 & & \\
\hline
\end{tabular}

Table 3: Predictive models obtained for each microorganism.

Microorganisms

\begin{tabular}{|c|c|c|c|c|c|}
\hline Coefficients & L. innосиа & S. aureus & E. faecalis & $P$. aeruginosa & S. cerevisiae \\
\hline$\beta_{1}$ & +6.16 & +6.48 & +6.33 & +1.55 & +6.37 \\
\hline$\beta_{2}$ & +6.15 & +6.48 & +6.33 & +4.17 & +0.40 \\
\hline$\beta_{3}$ & +6.06 & +6.48 & +6.10 & +6.26 & +6.35 \\
\hline$\beta_{12}$ & -6.83 & +0.00 & -4.48 & +2.31 & +11.98 \\
\hline$\beta_{13}$ & -0.026 & +0.00 & -0.035 & +10.34 & -0.10 \\
\hline$\beta_{23}$ & -0.029 & +0.00 & -0.035 & +4.47 & +11.94 \\
\hline$\beta_{123}$ & +24.16 & +0.00 & +18.34 & +33.39 & -21.83 \\
\hline
\end{tabular}

Table 4: Optimal antimicrobial combinations between the three AM agents.

\begin{tabular}{ccccccc}
\hline Run* & $\begin{array}{c}\text { Carvacrol } \\
(\boldsymbol{\%})\end{array}$ & $\begin{array}{c}\text { GSE } \\
(\boldsymbol{\%})\end{array}$ & $\begin{array}{c}\text { Chitosan } \\
(\boldsymbol{\%})\end{array}$ & $\begin{array}{c}\text { Carvacrol } \\
(\mathbf{p p m})\end{array}$ & $\begin{array}{c}\text { GSE } \\
(\mathbf{p p m})\end{array}$ & $\begin{array}{c}\text { Chitosan } \\
(\boldsymbol{\%} \mathbf{w} / \mathbf{v})\end{array}$ \\
\hline OAMC-1 & 3.2 & 34.2 & 62.6 & 9.6 & 684 & 1.25 \\
OAMC-2 & 20 & 20 & 60 & 60 & 400 & 1.2 \\
OAMC-3 & 30 & 8 & 62 & 90 & 160 & 1.24 \\
Control & 0 & 0 & 100 & 0 & 0 & 1.25 \\
\hline
\end{tabular}

* All runs were performed in triplicate. 
Effect of carvacrol, grape seed extract and chitosan mixtures on microorganisms and food $\mid 31$

Guarda et al. (2011) studied the minimal inhibitory concentration of carvacrol and thymol, and also found the synergistic effect between the two agents (based on the MIC) for L. innocua, S. aureus, E. coli, S. cerevisiae and A. Niger. The results showed that there was a synergistic effect between carvacrol $(C)$ and thymol $(T)$ for the growth inhibition of all microorganisms at all combinations of concentrations $(0.5 T-$ $0.5 C ; 0.25 T-0.75 C ; 0.75 T-0.25 C$, expressed in fractions) at $100 \%$ of the MIC's of the AM agents. Synergistic effect was only observed for yeast when carvacrol and thymol were in equal proportions. At $50 \%$ of the MIC, no synergistic effect was found for any of the microorganisms. The study of synergistic effects between different agents is very important for several reasons, such as the decrease of essential oils' concentration in food and high concentrations of essential oils may cause sensory changes (Chi, Zivanovic, \& Penfield, 2006).

\subsection{Antioxidant activity}

The free radical scavenging activity of GSE was evaluated by the decrease in the peak area of the DPPH radical at $517 \mathrm{~nm}$. Fig. 3(a) shows the radical scavenging activities of carvacrol, GSE and BHT at different concentrations. The amount of DPPH radical decreased much more in the presence of GSE and BHT than carvacrol. The radical scavenging activity of carvacrol, GSE and BHT ranges from $23.7 \pm 3.3 \%, 72.6 \pm 0.3 \%$ and $54.9 \pm 3.3 \%$, respectively at $25 \mathrm{ppm}$ concentration. Sharp increases in radical scavenging activity, with an increase in the concentration of GSE and BHT, were observed at 50 ppm concentration, giving $82.4 \pm 0.4 \%$ and $79.1 \pm 0.9 \%$, respectively. Similar results for GSE were obtained by Jayaprakasha et al. (2003) at $50 \mathrm{ppm}$ concentration (70\% the radical scavenging activity).

The AOX properties of GSE are primarily due to flavonoids that can perform scavenging action on free radicals, in this case DPPH. The presence of a hydroxyl functional group in the structure and its position on the ring of the flavonoid molecule determines the AOX capacity (Arora, Nair, \& Strasburg, 1998). Addition

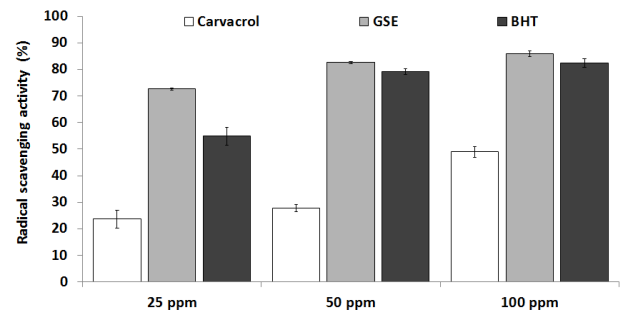

a

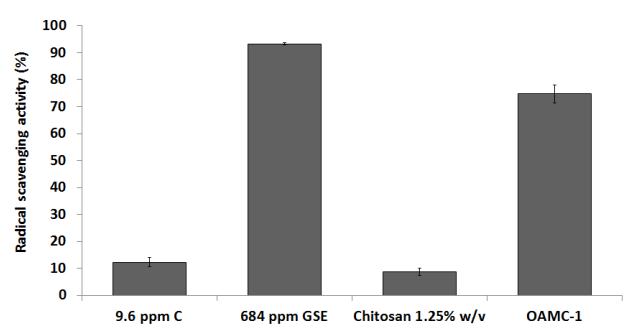

b
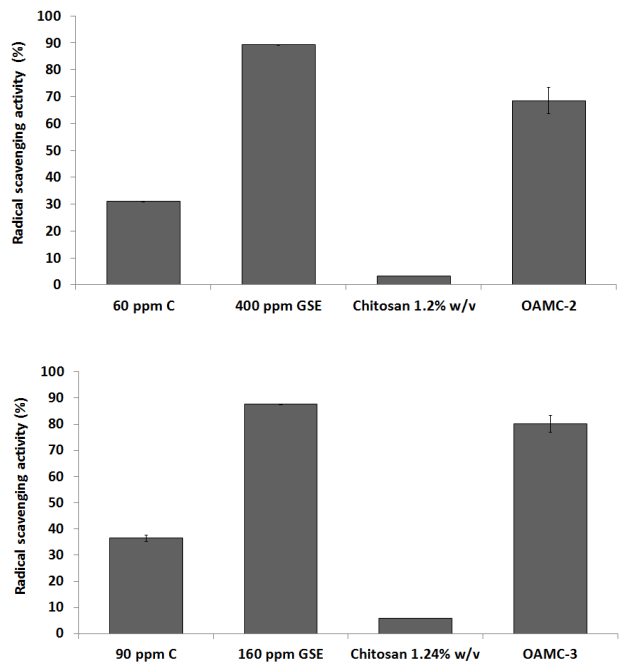

d

Figure 3: RSA of carvacrol, GSE and BHT at different concentrations (ppm) (a), RSA of agents at different concentrations of the OAMC-1 (b), OAMC-2 (c), and OAMC-3 (d) by the DPPH method. Error bars represent standard error. Different lower case letters for each concentrations are significantly different $(p<0.05)$. 
of hydroxyl groups to the flavonoid nucleus will enhance the AOX activity, while their substitution by methoxy groups diminishes the AOX activity (Majo, Guardia, Giammanco, Neve, \& Giammanco, 2008).

Amongst the different parts of a grape vine, grape seeds exhibit the highest AOX activity followed by the skin and the flesh (PastranaBonilla, Akoh, Sellappan, \& Krewer, 2003). The AOX potential of GSE is twenty and fifty fold greater than those of vitamins $\mathrm{E}$ and $\mathrm{C}$, respectively (Shi, Yu, Pohorly, \& Kakuda, 2003).

Fig. 3 shows the radical scavenging activities of carvacrol, GSE and chitosan with the optimal AM concentrations (OAMC-1, in Fig. 3(b); OAMC-2, in Fig. 3(c); and OAMC-3, in Fig. $3(\mathrm{~d})$ separately and in mixtures. An antagonistic effect of radical scavenging activity of GSE was observed in mixture with carvacrol and chitosan. This could be explained by hydrogen donating to chitosan and not to DPPH (Jayaprakasha et al., 2003).

Comparing the optimal $\mathrm{AM}$ combinations (OAMC-1, OAMC-2, OAMC-3), the best radical scavenging activity was found for OAMC3 ; therefore this mixture will be used in coating tests of the food matrices.

\subsection{Microbiological analysis}

The effect of coating with the OAMC-3 on the growth of $P$. aeruginosa and $S$. cerevisiae inoculated on the surface of fresh strawberries is shown in Fig. 4(a). Immediately after the coating application (by dipping and drying), the count of viable bacterial cells on the strawberries was reduced from $4.9 \log \mathrm{cfu}^{-1}$ of $P$. aeruginosa and about $4.8 \log \mathrm{cfu}^{-1}$ of $S$. cerevisiae on uncoated (control) strawberries to about $1.8 \log \mathrm{cfu}$ $\mathrm{g}^{-1}$ of $P$. aeruginosa and about $2.8 \log \mathrm{cfu}^{-1}$ of $S$. cerevisiae on coated strawberries, respectively. Park et al. (2005) studied the effect of chitosan coating with $0.3 \%$ potassium sorbate in strawberries and the results showed that immediately after coating application, Cladosporum sp showed a reduction of $1 \mathrm{log} \mathrm{cfu}^{-1}$.

After $48 \mathrm{~h}$ of the coating application, the level of viable cells on the strawberries was reduced from $4.2 \log \mathrm{cfu}^{-1}$ of $P$. aeruginosa and about 4.8 $\log \mathrm{cfu}^{-1}$ of $S$. cerevisiae on uncoated (control) strawberries to $\mathrm{a}<1.0 \log \mathrm{cfu}^{-1}$ of $P$. aeruginosa and about $3.3 \mathrm{log} \mathrm{cfu}^{-1}$ of $S$. cerevisiae on coated strawberries, respectively.

Fig. 5(a) shows visible contamination by mold growth on uncoated strawberries after 16 days, unlike the strawberries coated with OAMC-3 that presented no visible contamination. Nevertheless, comparing strawberries inoculated with $P$. aeruginosa and strawberries inoculated with $S$. cerevisiae, some mold growth could be observed in the latter. The effects of coating with the OAMC-3 on the growth of E. faecalis, L. innocua and $S$. aureus inoculated on the surface of fresh salmon are shown in Fig. 4(b). Immediately after the coating application (by dipping and drying), the level of viable bacterial cells in the salmon was reduced from $5.7 \log \mathrm{cfu} \mathrm{g}^{-1}$ of E. faecalis, about $6.2 \log \mathrm{cfu} \mathrm{g}^{-1}$ of L. innocua and about $5.4 \log \mathrm{cfu}^{-1}$ of $S$. aureus on uncoated (control) salmon to about $4.7 \log \mathrm{cfu} \mathrm{g}^{-1}$ of E. faecalis, about $4.1 \log \mathrm{cfu} \mathrm{g}^{-1}$ of L. innocua and about $5.2 \log \mathrm{cfu}^{-1}$ of $S$. aureus on coated salmon.

$48 \mathrm{~h}$ after the coating of salmon with OAMC-3, the level of viable cells was reduced from about $5.7 \log \mathrm{cfu} \mathrm{g}^{-1}$ of E. faecalis, about $6.2 \mathrm{log} \mathrm{cfu}$ $\mathrm{g}^{-1}$ of $L$. innocua and about $6.6 \mathrm{log} \mathrm{cfu}^{-1}$ of $S$. aureus on uncoated (control) salmon to about $4.7 \mathrm{log} \mathrm{cfu}^{-1}$ of E. faecalis, about $5.0 \mathrm{log} \mathrm{cfu}$ $\mathrm{g}^{-1}$ of L. innocua and about $5.9 \log \mathrm{cfu}^{-1}$ of $S$. aureus on coated salmon.

Fig. 5(b) shows visibly stronger decomposition of uncoated salmon with a yellow liquid draining after 14 days, while the salmon coated with OAMC-3 presented no such indications for any of the microorganisms studied.

The principal antibacterial compound found in the methanol extract from grape seed is gallic acid and it has shown inhibitory effects on E. coli and S. enteritidis (Shoko et al., 1999). The presence of gallic acid is responsible for their high affinity for the lipid bilayer, and it affects the membrane structure. The major phenolic constituents like epicatechin may alter the cell morphology by influencing the osmotic pressure of the cell, thus disrupting the cytoplasmic membrane and causing leakage of cell constituents (Perumalla \& Hettiarachchy, 2011).

Catechin is another compound with AM proper- 
Effect of carvacrol, grape seed extract and chitosan mixtures on microorganisms and food $\mid 33$

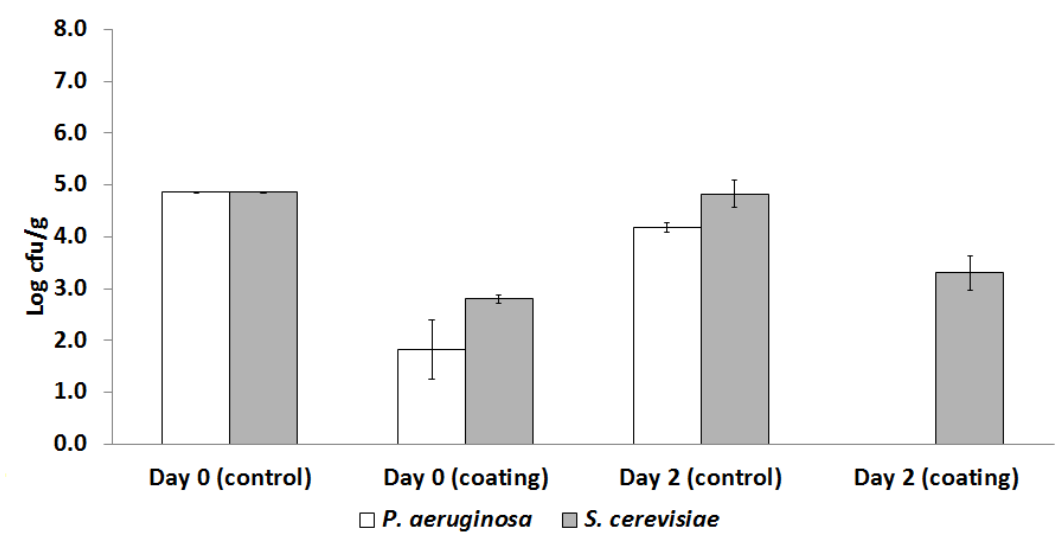

a

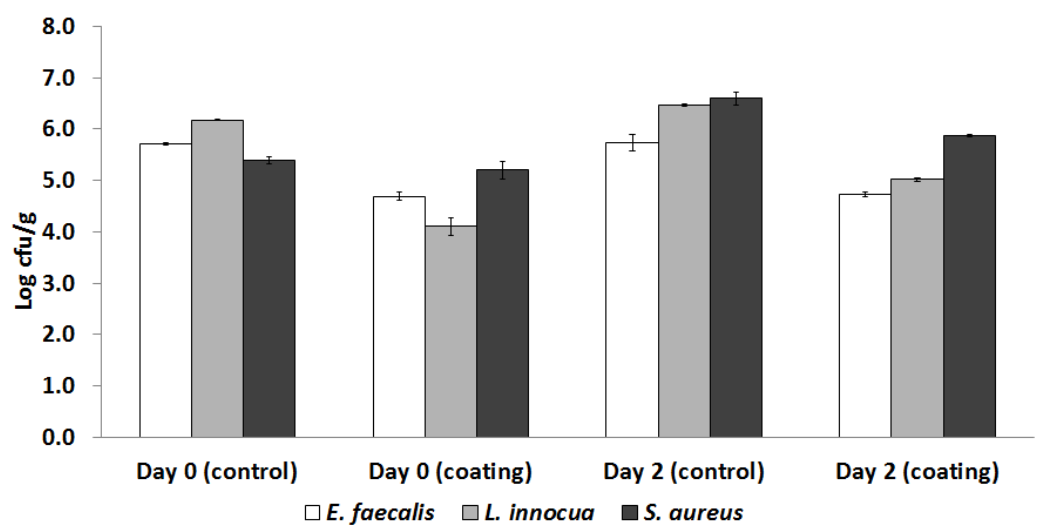

b

Figure 4: Effect of coating on strawberries upon P. aeruginosa and S. cerevisiae (a), and effect of coating on salmon upon E. faecalis, L. innocua, and S. aureus (b).

ties present in GSE. It can generate a deterioration effect on the lipid bi-layer membrane and as a result, produce a loss of the cell structure and function, leading to cell death (Perumalla \& Hettiarachchy, 2011).

Since most foods are mainly composed of water, carbohydrates, fats, proteins and $\mathrm{NaCl}$, it is important to know the influence of these components on the AM activity of any AM compounds. Devlieghere, Vermeulen, and Debevere (2004), determined the influence of different food components (starch, whey protein, $\mathrm{NaCl}$ and oil) on the AM effect of chitosan.

The results showed that the AM activity of chitosan was strongly decreased by high amounts of starch $(30 \% \mathrm{w} / \mathrm{v})$, leading to a significantly shorter lag phase and a significantly higher growth rate of Candida lambica. Furthermore, it was only mentioned that the gelatinized starch could interact negatively with the AM capacity of chitosan, but it is possible that native starch interacts in a different negative way on the AM capacity of chitosan. Similar results were obtained for proteins, due to decrease in the AM activity of chitosan when $10 \%$ of whey protein isolate was added. In the case of $\mathrm{NaCl}, 2 \%$ was enough to inhibit the AM activity of chitosan because it interferes with the electrostatic forces between chitosan and the microbial surface. An opposite effect with oil was observed, due to no 
34| Rubilar et al.
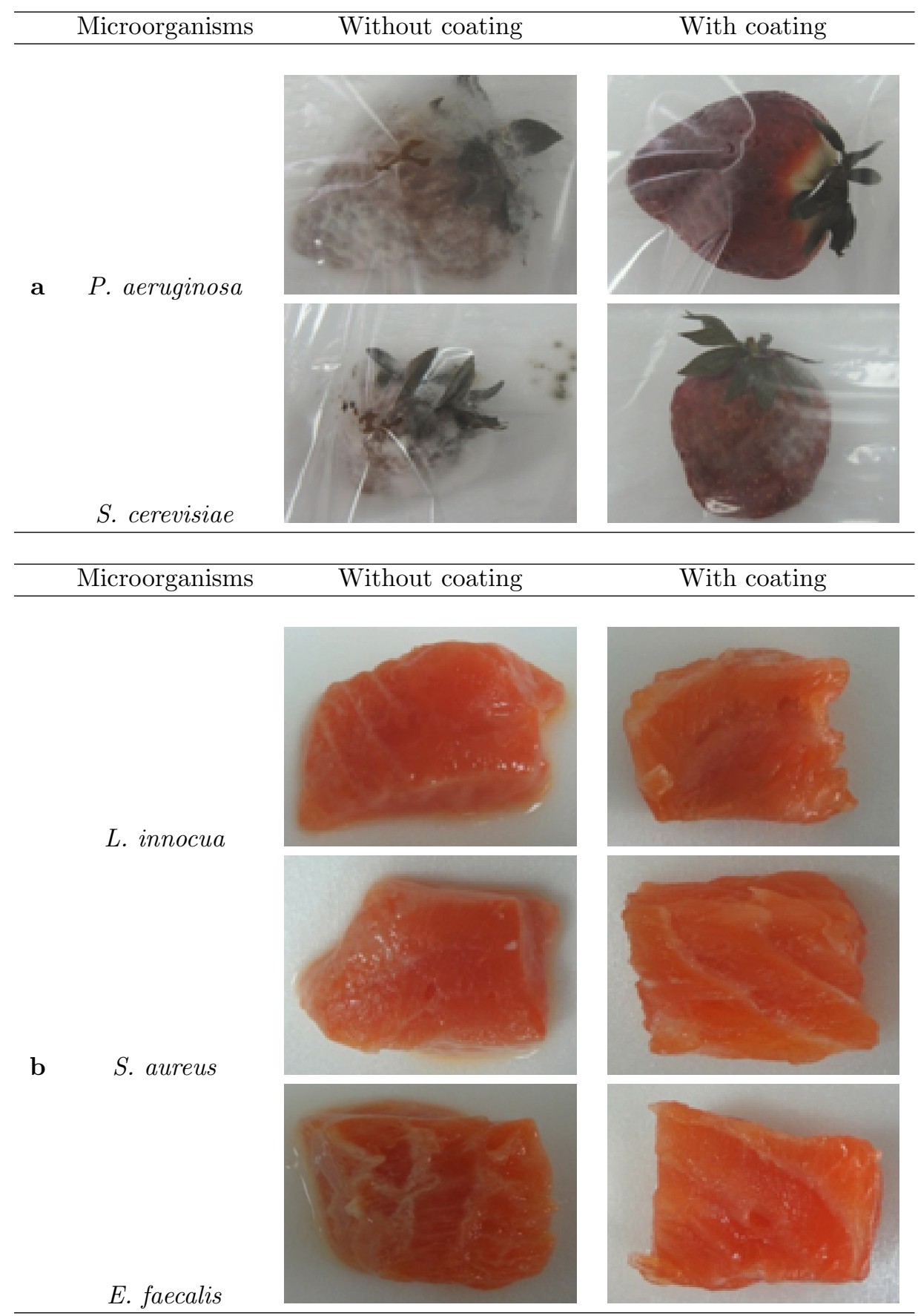

Figure 5: Strawberries inoculated with $P$. aeruginosa and S. cerevisiae, without and with coating, after 16 days (a), and salmon without and with coating, inoculated with $L$. innocua, S. aureus, and E. faecalis after 14 days (b). 
Effect of carvacrol, grape seed extract and chitosan mixtures on microorganisms and food $\mid 35$

influence in the AM capacity of chitosan. This phenomenon could be explained by the fact that chitosan is positioned at the outside of the emulsion drops due to the interaction between the positively charged chitosan and the negatively charged free fatty acid (Devlieghere et al., 2004; Jumaa \& Muller, 1999).

All these phenomena, might explain why the application of chitosan coatings on strawberries and salmon did not reduce six logarithmic for all microorganisms.

\section{Conclusions}

The natural AM agents (carvacrol and GSE) were found to be more effective against the studied gram-positive bacteria, as evidenced by their lower MIC values. The experimental validation confirmed that the optimal solutions obtained with carvacrol, GSE and chitosan inhibit at least six logarithmic of all microorganisms. GSE and OAMC-3 presented the highest AOX capacities. The coating with OAMC-3 presented significant log reduction on all microorganisms studied, from the beginning of the experiment and after $48 \mathrm{~h}$ of the coating application in strawberries and salmon. In the case of strawberries, $P$. aeruginosa was the microorganism more sensitive to the action of OAMC-3 than $S$. cerevisiae, while in the case of salmon, $S$. aureus was more resistant to the action of OAMC-3 than $E$. faecalis and L. innocua. For strawberries and salmon, the OAMC-3 did not reduce 6 logarithmic for all microorganisms (as well as in agar) because chitosan as a charged molecule could interact with charged molecules present in food, thereby decreasing significantly the AM capacity of the chitosan coating. The optimal AM mixture selected in this study can contribute to the development of active packaging with AM and AOX properties.

\section{Acknowledgements}

The author Javiera F. Rubilar gratefully acknowledges her Ph.D. grant Erasmus Mundus 2008-1022/001 Frame ECW/17 from the EACEA of the European Union. The author Rui M. S. Cruz would like to thank
Fundação para a Ciência e Tecnologia (Grant SFRH/BPD/70036/2010).

\section{References}

Aeschbach, R, Loliger, J, Scott, B., Murcia, A, Butler, J, Halliwell, B, \& Aruoma, O. (1994). Antioxidant actions of Thymol, Carvacrol, 6-Gingerol, Zingerone and Hydroxytyrosol. Food and Chemical Toxicology, 32(1), 31-36. doi:10.1016/02786915(84)90033-4

Anastasiadi, M., Chorianopoulos, N. G., Nychas, G.-J. E., \& Haroutounian, S. A. (2009). Antilisterial Activities of Polyphenol-Rich Extracts of Grapes and Vinification Byproducts. Journal of Agricultural and Food Chemistry, 57(2), 457-463. doi:10.1021/jf8024979

Arora, A, Nair, M., \& Strasburg, G. (1998). Structure-activity relationships for antioxidant activities of a series of flavonoids in a liposomal system. Free Radical Biology and Medicine, 24(9), 1355-1363. doi:10.1016/ S0891-5849(97)00458-9

Baydar, N., Ozkan, G, \& Sagdic, O. (2004). Total phenolic contents and antibacterial activities of grape (Vitis vinifera L.) extracts. Food Control, 15(5), 335-339. doi:10.1016/ S0956-7135(03)00083-5

Baydar, N. G., Sagdic, O., Ozkan, G., \& Cetin, S. (2006). Determination of antibacterial effects and total phenolic contents of grape (Vitis vinifera L.) seed extracts. International Journal of Food Science and Technology, 41(7), 799-804. doi:10.1111/j.13652621.2005.01095.x

Baydar, N. G., Ozkan, G., \& Yasar, S. (2007). Evaluation of the antiradical and antioxidant potential of grape extracts. Food Control, 18(9), 1131-1136. doi:10 . 1016/j . foodcont.2006.06.011

Burt, S. (2004). Essential oils: their antibacterial properties and potential applications in foods - a review. International Journal of Food Microbiology, 94(3), 223-253. doi:10. 1016/j.ijfoodmicro.2004.03.022

Chi, S, Zivanovic, S, \& Penfield, M. (2006). Application of chitosan films enriched with

IJFS April 2013 |Volume 2 pages 22-38 
oregano essential oil on Bologna - Active compounds and sensory attributes. Food Science and Technology International, 12(2), 111-117. doi:10 . 1177/ 1082013206063845

Corrales, M., Han, J. H., \& Tauscher, B. (2009). Antimicrobial properties of grape seed extracts and their effectiveness after incorporation into pea starch films. International Journal of Food Science and Technology, 44(2), 425-433. doi:10.1111/j.1365-2621. 2008.01790.x

Cosentino, S, Tuberoso, C., Pisano, B, Satta, M, Mascia, V, Arzedi, E, \& Palmas, F. (1999). In-vitro antimicrobial activity and chemical composition of Sardinian Thymus essential oils. Letters in Applied Microbiology, 29(2), 130-135. doi:10.1046/j.1472765X.1999.00605.x

Design-Expert software. (2005). Version 7.0.0. Stat-Ease, Minneapolis.

Devlieghere, F, Vermeulen, A, \& Debevere, J. (2004). Chitosan: antimicrobial activity, interactions with food components and applicability as a coating on fruit and vegetables. Food Microbiology, 21 (6), 703-714. doi:10.1016/j.fm.2004.02.008

Durango, A., Soares, N., Benevides, S, Teixeira, J, Carvalho, M, Wobeto, C, \& Andrade, N. (2006). Development and evaluation of an edible antimicrobial film based on yam starch and chitosan. Packaging Technology and Science, 19(1), 55-59. doi:10.1002/pts. 713

Fajardo, P., Martins, J. T., Fucinos, C., Pastrana, L., Teixeira, J. A., \& Vicente, A. A. (2010). Evaluation of a chitosan-based edible film as carrier of natamycin to improve the storability of Saloio cheese. Journal of Food Engineering, 101(4), 349-356. doi:10.1016/j.jfoodeng.2010.06.029

Geraldine, R. M., Ferreira Soares, N. d. F., Botrel, D. A., \& Goncalves, L. d. A. (2008). Characterization and effect of edible coatings on minimally processed garlic quality. Carbohydrate Polymers, 72(3), 403409. doi:10.1016/j.carbpol.2007.09.012

Guarda, A., Rubilar, J. F., Miltz, J., \& Galotto, M. J. (2011). The antimicrobial activity of microencapsulated thymol and carvacrol.
International Journal of Food Microbiology, 146(2), 144-150. doi:10.1016/ j . ijfoodmicro.2011.02.011

Hammer, K., Carson, C., \& Riley, T. (1999). Antimicrobial activity of essential oils and other plant extracts. Journal of Applied Microbiology, 86(6), 985-990. doi:10.1046/ j.1365-2672.1999.00780.x

Jayaprakasha, G., Selvi, T, \& Sakariah, K. (2003). Antibacterial and antioxidant activities of grape (Vitis vinifera) seed extracts. Food Research International, 36(2), 117-122. doi:10 . 1016 / S0963 - 9969(02) 00116-3

Jumaa, M, \& Muller, B. (1999). Physicochemical properties of chitosan-lipid emulsions and their stability during the autoclaving process. International Journal of Pharmaceutics, 183(2), 175-184. doi:10.1016/S03785173(99)00086-1

Jung, E. J., Youn, D. K., Lee, S. H., No, H. K., Ha, J. G., \& Prinyawiwatkul, W. (2010). Antibacterial activity of chitosans with different degrees of deacetylation and viscosities. International Journal of Food Science and Technology, 45(4), 676-682. doi:10 . 1111/j.1365-2621.2010.02186.x

Kim, K. W., \& Thomas, R. L. (2007). Antioxidative activity of chitosans with varying molecular weights. Food Chemistry, 101 (1), 308-313. doi:10.1016/j.foodchem. 2006.01 .038

Lambert, R., Skandamis, P., Coote, P., \& Nychas, G. (2001). A study of the minimum inhibitory concentration and mode of action of oregano essential oil, thymol and carvacrol. Journal of Applied Microbiology, 91 (3), 453-462. doi:10.1046/j.1365-2672. 2001.01428.x

Majo, D., Guardia, M., Giammanco, S., Neve, L., \& Giammanco, M. (2008). The antioxidant capacity of red wine in relationship with its polyphenolic constituents. Food Chemistry, 111 (1), 45-49.

Marino, M, Bersani, C, \& Comi, G. (2001). Impedance measurements to study the antimicrobial activity of essential oils from Lamiaceae and Compositae. International Journal of Food Microbiology, 67(3), 187195. doi:10.1016/S0168-1605(01)00447-0 
Effect of carvacrol, grape seed extract and chitosan mixtures on microorganisms and food $\mid 37$

Myers, R., \& Montgomery, D. (1995). Experiments with mixtures. In response Surface Methodology, Process and Product optimization Using Designed Experiments. pp. 535-623. New York: Wiley Interscience.

Ojagh, S. M., Rezaei, M., Razavi, S. H., \& Hosseini, S. M. H. (2010). Effect of chitosan coatings enriched with cinnamon oil on the quality of refrigerated rainbow trout. Food Chemistry, 120(1), 193-198. doi:10.1016/j. foodchem.2009.10.006

Park, S., \& Zhao, Y. (2004). Incorporation of a high concentration of mineral or vitamin into chitosan-based films. Journal of Agricultural and Food Chemistry, 52(7), 19331939. doi:10.1021/jf034612p

Park, S., Stan, S., Daeschel, M., \& Zhao, Y. (2005). Antifungal coatings on fresh strawberries (Fragaria $\mathrm{x}$ ananassa) to control mold growth during cold storage. Journal of Food Science, 70(4), M202-M207.

Pastrana-Bonilla, E, Akoh, C., Sellappan, S, \& Krewer, G. (2003). Phenolic content and antioxidant capacity of muscadine grapes. Journal of Agricultural and Food Chemistry, 51(18), 5497-5503. doi:10 . $1021 /$ jf030113c

Paulus, W. (1993). Directory of Microbicides for the Protection of Materials. A Handbook, pp. 496. The Netherlands: Springer.

Perumalla, A. V. S., \& Hettiarachchy, N. S. (2011). Green tea and grape seed extracts - Potential applications in food safety and quality. Food Research International, 44(4), 827-839. doi:10 . 1016 / j . foodres . 2011.01.022

Pintore, G, Usai, M, Bradesi, P, Juliano, C, Boatto, G, Tomi, F, ... Casanova, J. (2002). Chemical composition and antimicrobial activity of Rosmarinus officinalis L. oils from Sardinia and Corsica. Flavour and Fragrance Journal, 17(1), 15-19. doi:10. 1002/ffj.1022

Pranoto, Y, Rakshit, S., \& Salokhe, V. (2005). Enhancing antimicrobial activity of chitosan films by incorporating garlic oil, potassium sorbate and nisin. LWT-Food Science and Technology, 38(8), 859-865. doi:10.1016/j.lwt.2004.lwt.2004.09.014
Rabea, E., Badawy, M., Stevens, C., Smagghe, G, \& Steurbaut, W. (2003). Chitosan as antimicrobial agent: Applications and mode of action. Biomacromolecules, 4(6), 14571465. doi:10.1021/bm034130m

Rhodes, P., Mitchell, J., Wilson, M., \& Melton, L. (2006). Antilisterial activity of grape juice and grape extracts derived from Vitis vinifera variety Ribier. International Journal of Food Microbiology, 107(3), 281-286. doi:10.1016/j.ijfoodmicro.2005.10.022

Rodriguez Vaquero, M. J., Alberto, M. R., \& Manca de Nadra, M. C. (2007). Influence of phenolic compounds from wines on the growth of Listeria monocytogenes. Food Control, 18(5), 587-593. doi:10.1016/j . foodcont.2006.02.005

Scalbert, A. (1991). Antimicrobial Properties Of Tannins. Phytochemistry, 30(12), 38753883. doi:10.1016/0031-9422(91)83426-L

Šegvić-Klarić, M., Kosalec, I., Mastelić, J., Piecková, E., \& Pepeljnak, S. (2006). Antifungal activity of thyme (Thymus vulgaris $L$.) essential oil and thymol against moulds from damp dwellings. Letters in Applied Microbiology, 44 (1), 36-42.

Shi, J., Yu, J., Pohorly, J., \& Kakuda, Y. (2003). Polyphenolics in grape seeds-biochemistry and functionality. Journal of Medicinal Food, 6(4), 291-299.

Shoko, T., Soichi, T., Megumi, M., Eri, F., Jun, K., \& Michiko, W. (1999). Isolation and identification of an antibacterial compound from grape and its application to foods. Nippon Nogeikagaku Kaishi, 73(2), 125128.

Sivropoulou, A, Papanikolaou, E, Nikolaou, C, Kokkini, S, Lanaras, T, \& Arsenakis, M. (1996). Antimicrobial and cytotoxic activities of Origanum essential oils. Journal of Agricultural and Food Chemistry, 44 (5), 1202-1205. doi:10.1021/jf950540t

Smith-Palmer, A, Stewart, J, \& Fyfe, L. (1998). Antimicrobial properties of plant essential oils and essences against five important food-borne pathogens. Letters in Applied Microbiology, 26(2), 118-122.

Sokmen, A, Gulluce, M, Akpulat, H., Daferera, D, Tepe, B, Polissiou, M, ... Sahin, F. (2004). The in vitro antimicrobial and 
antioxidant activities of the essential oils and methanol extracts of endemic Thymus spathulifolius. Food Control, 15(8), 627634. doi:10.1016/j.foodcont.2003.10.005

Statgraphics Centurion XV software. (2006). Version 15.2. 05. Statistical graphics corp. Warrenton, Virginia.

Thuille, N, Fille, M, \& Nagl, M. (2003). Bactericidal activity of herbal extracts. International Journal of Hygiene and Environmental Health, 206(3), 217-221. doi:10 . 1078/1438-4639-00217

US Food and Drug Administration. (2006). Database of select committee on GRAS substances (SCOGS) Reviews.

Vaara, M. (1992). Agents that increase the permeability of the Outer-MembranE. Microbiological Reviews, 56(3), 395-411.

Veldhuizen, E., Tjeerdsma-Van Bokhoven, J., Zweijtzer, C, Burt, S., \& Haagsman, H. (2006). Structural requirements for the antimicrobial activity of carvacrol. Journal of Agricultural and Food Chemistry, 54(5), 1874-1879. doi:10.1021/jf052564y

Xia, E.-Q., Deng, G.-F., Guo, Y.-J., \& Li, H.B. (2010). Biological Activities of Polyphenols from Grapes. International Journal of Molecular Sciences, 11(2), 622-646. doi:10. 3390/ijms11020622 